\title{
Some Developments of the Weak Stellar Magnetic Field Determination Method for the Example of Cygnus X-1
}

\author{
N. G. Bochkarev ${ }^{1}$ and E. A. Karitskaya ${ }^{2}$ \\ ${ }^{1}$ Sternberg Astron. Inst., Universitetskij prosp. 13, 119991 Moscow, Russia \\ email: boch@sai.msu.ru \\ ${ }^{2}$ Astronomical Institute of RAS, Pyatnitskaya str. 48, 119017 Moscow, Russia \\ email: karitsk@sai.msu.ru
}

\begin{abstract}
Some developments of measurements of the weak stellar magnetic fields by the least square technique applied to spectropolarimetric data are proposed and used for the X-ray binary Cyg X-1 = HDE 226868 (the optical counterpart is an O 9.7 supergiant).
\end{abstract}

Keywords. Stars: magnetic fields, techniques: polarimetric, techniques: spectroscopic, methods: statistical, stars: early-type, stars: individual (Cyg X-1 = HDE $226868=$ V1357 Cyg), (stars:) supergiants, (ISM:) extinction, X-rays: binaries, black hole physics

In contrast to the stars with strong magnetic fields (mainly $\mathrm{A}$ and late B types), luminous O-stars have usually weaker field and significant interstellar / circumstellar linear polarization (up to $\sim 10 \%$ ).

Any spectropolarimeter has cross-talk between linear and circular polarization within the instrument. It creates a spurious circularly polarized wavelength-dependent continual component of radiation for stars with linear polarization.

As a result, more and more often targets for magnetic field measurements have spectra of Stokes parameter $V$ (measuring circular polarization) and spectra of ratios $V / I$ ( $I$ is Stokes parameter for total intensity) containing wavelength-dependent continual components $C_{V}(\lambda)$ and $C_{V / I}(\lambda)$, where $\lambda$ is the wavelength.

Bochkarev \& Karitskaya (2011) proposed some developments of measurements of the weak stellar magnetic fields by the least square technique applied to spectropolarimetric data and used them for the X-ray binary Cyg X-1 = HDE 226868 (the optical counterpart is an O 9.7 supergiant).

The parameters of the object are: magnitude $m_{V}=9^{m} ;>95 \%$ of optical radiation is produced by the O9.7 Iab star; interstellar extinction $A_{V}=3.36^{m}$; interstellar/circumstellar linear polarization $\sim 5 \%$; stellar wind $M_{\text {dot }} \sim(2-3) * 10^{-6} M_{\text {sun }} / \mathrm{yr}$; chemical peculiarities (mainly He, N, Si excess); and rotation velocity $V \sin i=95 \mathrm{~km} / \mathrm{s}$.

Our observations were made at the Very Large Telescope (VLT) $8.2 \mathrm{~m}$ (Mount Paranal, Chile) in the spectropolarimetric mode of the FORS1 spectrograph with resolution $R=$ 4000 in the range $3680-5129 \AA$, with signal-to-noise ratio $S / N=1500-3500$ (for $I$ ) from June 18 to July 9, 2007 and from July 14 to July 30, 2008 (Cyg X-1 in hard X-ray state). A total of 13 spectra of intensity $I$ and circular polarization $V$ were obtained (Karitskaya et al. 2009, Karitskaya et al. 2010).

The mean longitudinal magnetic field $\left\langle B_{z}\right\rangle$ was determined by statistical processing of the $V(\lambda)$ and $I(\lambda)$ spectra using the equation: $\frac{V}{I}=-C_{Z} g_{\text {eff }} \lambda^{2} \frac{1}{I} \frac{\mathrm{d} I}{\mathrm{~d} \lambda}\left\langle B_{z}\right\rangle+\frac{V_{0}}{I_{0}}$ (e.g., Landstreet 1982), where $g_{\text {eff }}$ is the effective Lande factor, $C_{Z}=\frac{e}{4 \pi m_{e} c^{2}}=4.67 * 10^{-13} \stackrel{\circ}{A}^{-1} G^{-1}$, 
$\frac{V_{0}}{I_{0}}=$ const. The least squares method is used for the $\left\langle B_{z}\right\rangle$ calculation (e.g., Hubrig et al. 2004).

The sources of noise in the $\left\langle B_{z}\right\rangle$ measurements, which should be removed from the $I$ and $V / I$-spectra, are: 1) interstellar lines and narrow diffuse interstellar bands (DIBs); 2) defects (including residual cosmic ray tracks not removed by the standard observation processing); 3) He II $4686 \AA$ line with profile including the accretion-structure emission component; and 4) the emission components of the lines showing the P Cyg effect. In addition, we removed some $\lambda$ intervals containing noise only. We found no pollution by telluric lines in our spectra.

The slope value $S=\mathrm{d} C_{V / I} / \mathrm{d} \lambda \sim 10^{-6} \AA^{-1}$ varied irregularly from night to night. The most probable reason for the $V / I$-spectra sloping is the cross-talk between linear and circular polarization within the FORS1 analyzing equipment.

The application of the least squares method without correcting for the $V / I$ trend results in distorted or even spurious $\left\langle B_{z}\right\rangle$ values and in a distorted accuracy of these values. That happens for at least 2 reasons: 1) strong violation of Gauss statistics by the residuals; 2) appearance of $\left\langle B_{z}\right\rangle$ spurious component $\propto\left(\Delta \lambda_{D} / \lambda\right)^{2} * \mathrm{~d} C_{V / I} / \mathrm{d} \lambda$. Here $\Delta \lambda_{D}$ is the spectral line width.

For our Cyg X-1 FORS1 observations, spurious $\left\langle B_{z}\right\rangle$ from single spectral line and sloped $V / I$ continuum without any Zeeman feature is several Gauss. To avoid any influence of the $V / I$-continuum slope on $\left\langle B_{z}\right\rangle$ measurements, we subtracted the linear trends from $V / I$ spectra. In the case of our Cyg X-1 observations, the uncorrected slopes of the $\mathrm{V} / \mathrm{I}$-spectra create $\left\langle B_{z}\right\rangle$ shifts from -20 to $-84 \mathrm{G}$ (Bochkarev \& Karitskaya 2011).

We normalized the $I$-spectra using a pseudo-continuum. The wavelength dependence of the $I$-continuum $C_{I}(\lambda)$ is produced by: the source energy distribution, interstellar reddening, broad DIBs, atmospheric extinction, and the sensitivity of the detector. The $I$-spectrum slopes reach $\mid \mathrm{d}(\log (I(\lambda)) / \mathrm{d}(\log (\lambda)) \mid \sim 20$. The removal of the slope gives a $\left\langle B_{z}\right\rangle$ correction up to $\sim 20 \mathrm{G}$. It is usually less than the statistical errors $\sigma\left(\left\langle B_{z}\right\rangle\right)$, which are $\geqslant 20-30 \mathrm{G}$.

The value of the mean longitudinal magnetic field in the optical component (O 9.7 Iab supergiant) changes regularly with the orbital phase, and reaches a maximum of $130 \mathrm{G}$ $(\sigma \approx 20 \mathrm{G})$ (Karitskaya et al. 2009, 2010).

The measurements based on the Zeeman effect were carried out over all the observed supergiant photosphere absorption spectral lines. Similar measurements over the emission line He II $\lambda 4686 \AA$ yielded a value of several hundred Gauss with a smaller significance level. The emission component of this line originates in the outer parts of the accretion structure. So we measure $\left\langle B_{z}\right\rangle$ in this region (Karitskaya et al. 2009, 2010).

We got $\left\langle B_{z}\right\rangle \sim 100 \mathrm{G}$ for the star's photosphere. The gas stream carries the field on to the accretion structure; the gas is compressed by interaction with the structure's outer rim. Gas density is increased by a factor of 6-10 (we obtained several hundred Gauss). According to the Shakura - Sunyaev magnetized accretion disc model at $3 R_{g}: B \sim 10^{9} \mathrm{G}$. Taking into account radiative pressure predominance inside $\sim 10-20 R_{g}$, we get: $B\left(3 R_{g}\right)$ $\sim(2-3) 10^{8}$ G. Such magnetic fields can be responsible for the observed X-ray flickering.

This study was supported by RFBR grant 09-02-01136.

\section{References}

Bochkarev, N. G. \& Karitskaya, E. A. 2011, in D. O. Kudryavtsev, \& I. I. Romanyuk (eds.), Magnetic Stars (N.Arkhyz: SAO RAS publ.), p. 199

Hubrig, S., Szeifert, T., Schöller, M. et al., 2004, A\&A, 415, 685

Karitskaya, E. A., Bochkarev, N. G., Hubrig, S. et al., 2009, astro-ph 0908.2719

Karitskaya, E. A., Bochkarev, N. G., Hubrig, S. et al., 2010, Inf. Bull. Variable Stars, 5950,1

Landstreet J. D. 1982, ApJ, 258, 639 